\title{
Characterisation of Frenet-Serret and Bishop Motions with applications to Needle Steering
}

\author{
J.M. Selig \\ Faculty of Business, \\ London South Bank University, U.K.
}

\begin{abstract}
Frenet-Serret and Bishop rigid-body motions have many potential applications in robotics, graphics and computer aided design. In order to study these motions new characterisations in terms of their velocity twists are derived. This is extended to general motions based on any moving frame to a space curve. Further it is shown that any such general moving frame motion is the product of a Frenet-Serret motion with a rotation about the tangent vector.

These ideas are applied to a simple model of needle steering. A simple kinematic model of the path of the needle is derived. It is then shown that this leads to Frenet-Serret motions of the needle tip but with constant curvature. Finally some remarks about curves with constant curvature are made.
\end{abstract}




\section{Introduction}

\section{$1.1 \quad$ Background}

Frenet-Serret motions are the motions traced by the Frenet frame of a curve in space. Likewise Bishop motions are produced by following the Bishop frame of a curve. Klok ${ }^{1}$ investigated these motions in order to produce sweeping surfaces from curves. It was probably Wagner and Ravani, ${ }^{2}$ who first suggested using rational Frenet-Serret motions for motion interpolation. Although the notion of Frenet-Serret motions themselves have a much longer history, see Bottema and Roth, ${ }^{3}$ for example. Frenet-Serret motions occur in other situations too. In medical applications a flexible needle can be steered through soft tissue. As discussed in Duindam et $a{ }^{4}{ }^{4}$ the motion of the needle will be a special FrenetSerret motion, see also section 3 below.

In a seminal paper Bishop ${ }^{5}$ explored different ways to frame curves and defined an alternative to the standard Frenet frame which has many useful properties. Bishop's frame for a curve is also sometimes called the natural frame or the rotation-minimising frame. The motion associated with the Bishop frame of a curve has been suggested by several workers for different applications. ${ }^{1,6,7}$ Notice that any regular curve will define a Frenet-Serret or Bishop motion for a rigid body.

Previous studies have concentrated on the curve defining the motion. For example, Wagner and Ravani's approach was to find space curves with rational Frenet-Serret motions. They showed that the condition for a curve to have a rational Frenet-Serret motion is that its speed and curvature functions must be rational functions of the arc-length parameter. The problem of finding rational Bishop motions has been addressed in the the work of Farouki et al. ${ }^{8}$ The focus there was on finding space curves, Pythagorean-hodographs, with the appropriate properties. 
A different problem is studied here. The question considered first is: Given a rigid-body motion, how can one tell if it is a Frenet-Serret or a Bishop motion, and if it is what is the underlying curve in space for the motion? The conditions for a rigid-motion to be a Frenet-Serret or a Bishop motion turn out to be that the velocity twist of the body, computed in a coordinate frame fixed in the body, must lie in a particular screw system.

\section{Rigid Body Motions Determined by Space Curves}

\subsection{Motivation}

Let $\mathbf{p}(t)$ be a curve in space parametrised by $t$. Such a curve determines a rigidbody motion as follows. At each parameter value take the origin of the frame fixed in the moving body as $\mathbf{p}(t)$ and orient this frame so that the $x, y$ and $z$ axes in the moving body lie along the tangent, normal and binormal vectors of the Frenet frame to the curve. This is the definition of the well known Frenet-Serret motion. ${ }^{3}$ As a $4 \times 4$ matrix such a motion can be written as,

$$
G(t)=\left(\begin{array}{cc}
R(t) & \mathbf{p}(t) \\
0 & 1
\end{array}\right),
$$

where the rotation matrix $R$ is given by,

$$
R(t)=(\mathbf{t}|\mathbf{n}| \mathbf{b})
$$

and $\mathbf{t}, \mathbf{n}$ and $\mathbf{b}$ are respectively the tangent, normal and binormal vectors to the curve.

To motivate the discussion here consider the following rigid-body motion,

$$
M(t)=\left(\begin{array}{cccc}
\frac{1-t^{2}}{\sqrt{2}\left(1+t^{2}\right)} & \frac{-2 t}{\left(1+t^{2}\right)} & \frac{-\left(1-t^{2}\right)}{\sqrt{2}\left(1+t^{2}\right)} & \frac{-\sqrt{2} t^{5}+2 \sqrt{2} t^{3}+3 t^{2}+3 \sqrt{2} t-3}{3 \sqrt{2}\left(1+t^{2}\right)} \\
\frac{2 t}{\sqrt{2}\left(1+t^{2}\right)} & \frac{\left(1-t^{2}\right)}{\left(1+t^{2}\right)} & \frac{-2 t}{\sqrt{2}\left(1+t^{2}\right)} & \frac{t^{4}+t^{2}-\sqrt{2} t}{\left(1+t^{2}\right)} \\
\frac{1}{\sqrt{2}} & 0 & \frac{1}{\sqrt{2}} & \frac{1}{3} t^{3}+t+\frac{1}{\sqrt{2}} \\
0 & 0 & 0 & 1
\end{array}\right) .
$$


Now consider the question: Is this a Frenet-Serret motion as described above? The definition of a Frenet-Serret motion begins with a curve in space, here we have no particular curve in space. However under the action of this motion every point in space will trace out a trajectory. So perhaps the question should be sharpened to: Is there a point in space where the Frenet frame for the point's trajectory coincides with the given motion?

It might be argued that there is a particular point distinguished by the motion given above, the origin of the coordinates. This point traces the trajectory,

$$
\left(\begin{array}{l}
x(t) \\
y(t) \\
z(t)
\end{array}\right)=\left(\begin{array}{c}
\frac{-\sqrt{2} t^{5}+2 \sqrt{2} t^{3}+3 t^{2}+3 \sqrt{2} t-3}{3 \sqrt{2}\left(1+t^{2}\right)} \\
\frac{t^{4}+t^{2}-\sqrt{2} t}{\left(1+t^{2}\right)} \\
\frac{1}{3} t^{3}+t+\frac{1}{\sqrt{2}}
\end{array}\right) .
$$

The tangent to this curve is given by,

$$
\mathbf{t}=\left(\begin{array}{c}
\frac{1+2 \sqrt{2} t+t^{2}-t^{4}-t^{6}}{\left(1+t^{2}\right) \sqrt{4+8 t^{2}+12 t^{4}+8 t^{6}+2 t^{8}}} \\
\frac{-\sqrt{2}+2 t+\sqrt{2} t^{2}+4 t^{3}+2 t^{5}}{\left(1+t^{2}\right) \sqrt{4+8 t^{2}+12 t^{4}+8 t^{6}+2 t^{8}}} \\
\frac{\left(1+t^{2}\right)^{2}}{\sqrt{4+8 t^{2}+12 t^{4}+8 t^{6}+2 t^{8}}}
\end{array}\right) .
$$

It is clear that this is different from the first column of the matrix $M(t)$ and hence the motion is not a Frenet-Serret motion about this trajectory. But there remains the question: Is it a Frenet-Serret motion for the trajectory of some other point, and if so, which point?

\subsection{Frenet-Serret Motion}

In general, given an arbitrary rigid-body motion how could one determine whether or not it was such a Frenet-Serret motion? To answer this question neatly it is useful to make a small definition. It is well known that the Frenet frame of a curve does not exist at points where the speed or the curvature vanishes. It is usual to talk about regular curves, whose speed is never zero. We also want to exclude from discussion curves with points of zero curvature. Hence the small definition needed is that here a regular Frenet-Serret motion will be taken 
to mean a Frenet-Serret motion based on a regular curve with non-vanishing curvature. With this definition it is possible to state the main theorem of this section:

Theorem 2.1 A rigid-body motion is a regular Frenet-Serret motion if and only if its body-frame velocity twist lies in a $I B_{0}$ three-system of screws with moduli $p_{a}=p_{b}=0$, with non-zero velocity in the translational direction.

Proof Consider the body-frame velocity twist, this is given by,

$$
S_{B}=G^{-1} \frac{d}{d t} G .
$$

The derivative of the matrix $G$ can be found using the famous Frenet-Serret equations for the curve:

$$
\begin{aligned}
& \dot{\mathbf{t}}=\quad \nu \kappa \mathbf{n}, \\
& \dot{\mathbf{n}}=-\nu \kappa \mathbf{t} \quad+\nu \tau \mathbf{b} \\
& \dot{\mathbf{b}}=-\nu \tau \mathbf{n},
\end{aligned}
$$

together with the definition of the tangent vector,

$$
\dot{\mathbf{p}}=\nu \mathbf{t}
$$

In the above $\nu$ is the speed of the curve since an arbitrary parametrisation of the curve has been assumed. That is, the parametrisation is not necessarily a unit speed parametrisation. The functions $\kappa$ and $\tau$ are the usual curvature and torsion functions of the curve. The above is possible provided the speed $\nu$ and curvature $\kappa$ do not vanish.

This gives,

$$
\frac{d}{d t} G=\nu G\left(\begin{array}{cccc}
0 & -\kappa & 0 & 1 \\
\kappa & 0 & -\tau & 0 \\
0 & \tau & 0 & 0 \\
0 & 0 & 0 & 0
\end{array}\right)
$$


and hence,

$$
S_{B}=\nu\left(\begin{array}{cccc}
0 & -\kappa & 0 & 1 \\
\kappa & 0 & -\tau & 0 \\
0 & \tau & 0 & 0 \\
0 & 0 & 0 & 0
\end{array}\right) \text { or as a twist vector, } \mathbf{s}_{B}=\nu\left(\begin{array}{c}
\tau \\
0 \\
\kappa \\
1 \\
0 \\
0
\end{array}\right)
$$

As $\nu, \kappa$ and $\tau$ vary along the curve it is clear that this twist is a linear combination of three constant twists,

$$
\mathbf{s}_{1}=\left(\begin{array}{l}
1 \\
0 \\
0 \\
0 \\
0 \\
0
\end{array}\right), \quad \mathbf{s}_{3}=\left(\begin{array}{l}
0 \\
0 \\
1 \\
0 \\
0 \\
0
\end{array}\right) \text { and } \mathbf{s}_{4}=\left(\begin{array}{l}
0 \\
0 \\
0 \\
1 \\
0 \\
0
\end{array}\right)
$$

That is, $\mathbf{s}_{B}$ lies in a three-system of screws.

In the appendix it is shown that this is a $I B_{0}$ system with moduli $p_{a}=p_{b}=0$. The coefficient of the translational twist $\mathbf{s}_{4}$ is simply the speed $\nu$ and this was assumed to be non-zero.

On the other hand suppose that the twist velocity of a rigid-body motion was such that it always lay in a $I B_{0}$ three system with moduli $p_{a}=p_{b}=0$. Then, by the classification result of Gibson and Hunt, it would be possible to find a coordinate frame in the body such that the normal form for the screw system consisted of the twists $\mathbf{s}_{1}, \mathbf{s}_{3}$ and $\mathbf{s}_{4}$ above. That is, there would be a coordinate frame in the body such that the twist velocity could be written in the form,

$$
\mathbf{s}_{B}=\alpha \mathbf{s}_{1}+\beta \mathbf{s}_{3}+\gamma \mathbf{s}_{4} .
$$

The functions $\alpha, \beta$ and $\gamma$ can obviously be related to the speed, curvature and 
torsion of a Frenet-Serret motion; $\nu=\gamma, \kappa=\beta / \gamma$ and $\tau=\alpha / \gamma$. Notice that the orientation of this frame must be chosen so that $\kappa=\beta / \gamma$ is positive since the curvature of space curves is always positive. Note also that by the hypothesis of the theorem $\gamma$, the coefficient of the translational twist, is non-zero.

Finally here notice that the curve producing the Frenet-Serret motion is the trajectory of the origin of the frame giving the normal form of the screw system in the body.

\subsection{Example}

Returning to the motivating example given in section 2.1 above.

Using the results above it is a straightforward matter to verify that this motion is indeed a Frenet-Serret motion. All that is needed is to compute the body-fixed velocity twist of the motion. After some algebra the result is,

$$
S_{B}=M^{-1}(t) \frac{d}{d t} M(t)=\left(\begin{array}{cccc}
0 & \frac{-\sqrt{2}}{\left(1+t^{2}\right)} & 0 & \sqrt{2}\left(1+t^{2}\right) \\
\frac{\sqrt{2}}{\left(1+t^{2}\right)} & 0 & \frac{-\sqrt{2}}{\left(1+t^{2}\right)} & \frac{-\sqrt{2}}{\left(1+t^{2}\right)} \\
0 & \frac{\sqrt{2}}{\left(1+t^{2}\right)} & 0 & 0 \\
0 & 0 & 0 & 0
\end{array}\right) .
$$

This can be written as a six-dimensional vector as,

$$
\mathbf{s}_{B}=\left(\begin{array}{c}
\sqrt{2} /\left(1+t^{2}\right) \\
0 \\
\sqrt{2} /\left(1+t^{2}\right) \\
\sqrt{2}\left(1+t^{2}\right) \\
-\sqrt{2} /\left(1+t^{2}\right) \\
0
\end{array}\right) .
$$

This twist is not in the same form as the one given in (3) above. However, it is easy to see that we can transform it into the required form with a simple translation in the $z$-direction. In the adjoint representation a translation in the 
$z$-direction is given by a $6 \times 6$ matrix of the form,

$$
H_{z}=\left(\begin{array}{cc}
I_{3} & 0 \\
T_{z} & I_{3}
\end{array}\right)
$$

where $I_{3}$ is the $3 \times 3$ identity matrix. The required translation is by one unit in the $z$-direction and this is represented by,

$$
T_{z}=\left(\begin{array}{ccc}
0 & -1 & 0 \\
1 & 0 & 0 \\
0 & 0 & 0
\end{array}\right) .
$$

Thinking of this as a change in coordinates, the twist $\mathbf{s}_{B}$ in the new coordinates will be given by,

$$
H_{z} \mathbf{s}_{B}=\left(\begin{array}{c}
\sqrt{2} /\left(1+t^{2}\right) \\
0 \\
\sqrt{2} /\left(1+t^{2}\right) \\
\sqrt{2}\left(1+t^{2}\right) \\
0 \\
0
\end{array}\right) .
$$

Hence we can see, by the theorem above, that this is indeed a Frenet-Serret motion, generated by the curve traced by the origin in these new coordinates.

In the standard $4 \times 4$ representation of $S E(3)$ this coordinate change corresponds to a matrix,

$$
M_{z}=\left(\begin{array}{cccc}
1 & 0 & 0 & 0 \\
0 & 1 & 0 & 0 \\
0 & 0 & 1 & -1 \\
0 & 0 & 0 & 1
\end{array}\right) .
$$

So in the new coordinates the motion is given by,

$$
G(t)=M(t) M_{z}=\left(\begin{array}{cccc}
\frac{1-t^{2}}{\sqrt{2}\left(1+t^{2}\right)} & \frac{-2 t}{\left(1+t^{2}\right)} & \frac{-\left(1-t^{2}\right)}{\sqrt{2}\left(1+t^{2}\right)} & t-t^{3} / 3 \\
\frac{2 t}{\sqrt{2}\left(1+t^{2}\right)} & \frac{\left(1-t^{2}\right)}{\left(1+t^{2}\right)} & \frac{-2 t}{\sqrt{2}\left(1+t^{2}\right)} & t^{2} \\
\frac{1}{\sqrt{2}} & 0 & \frac{1}{\sqrt{2}} & t+t^{3} / 3 \\
0 & 0 & 0 & 1
\end{array}\right) .
$$


This motion can be recognised as the rational Frenet-Serret motion given by Wagner and Ravani. ${ }^{2}$ In these coordinates the curve traced by the origin, and hence the one which generates the motion, is given by the twisted cubic,

$$
\mathbf{p}(t)=\left(\begin{array}{c}
t-t^{3} / 3 \\
t^{2} \\
t+t^{3} / 3
\end{array}\right) .
$$

The speed, curvature and torsion of this curve are given by,

$$
\nu=\sqrt{2}\left(1+t^{2}\right), \quad \kappa=\frac{1}{\left(1+t^{2}\right)^{2}}, \quad \tau=\frac{1}{\left(1+t^{2}\right)^{2}} .
$$

Notice that the trajectory of a general point in space, not the origin, will be a curve of degree 5 . The motion does not pass through the identity in the group.

\subsection{Bishop Motion}

Bishop motion can be treated in a similar fashion to the Frenet-Serret motions. However, there is a slight difference between the Bishop frame and the Frenet frame. The Frenet frame is uniquely defined for any regular curve with nonzero curvature, by contrast the Bishop frame has some freedom. This freedom is removed by choosing a starting frame, effectively this can be done by selecting a vector in the normal plane to the curve at some point.

The Bishop frame exists even if the curvature functions $k_{1}$ or $k_{2}$ vanish. So here it is simple to define a regular Bishop motion as a Bishop motion based on a regular curve. Again these motions can be characterised by the screw system in which their velocity twist lies:

Theorem 2.2 A rigid-body motion is a regular Bishop motion if and only if its body-frame velocity twist lies in a IIB three-system of screws with moduli $p=0$, with non-zero translational velocity. 
Proof The equations for the Bishop frame are given by,

$$
\begin{aligned}
\dot{\mathbf{t}} & =\nu k_{1} \mathbf{n}_{1}+\nu k_{2} \mathbf{n}_{2}, \\
\dot{\mathbf{n}}_{1} & =-\nu k_{1} \mathbf{t}, \\
\dot{\mathbf{n}}_{2} & =-\nu k_{2} \mathbf{t} .
\end{aligned}
$$

In the above $\mathbf{n}_{1}$ and $\mathbf{n}_{2}$ are two mutually orthogonal normal vectors to the curve and $k_{1}$ and $k_{2}$ are two curvature-like functions. The motion is given by the $4 \times 4$ matrix,

$$
G(t)=\left(\begin{array}{cc}
R(t) & \mathbf{p}(t) \\
0 & 1
\end{array}\right)
$$

as above but now the rotation matrix $R$ is given by,

$$
R(t)=\left(\mathbf{t}\left|\mathbf{n}_{1}\right| \mathbf{n}_{2}\right) .
$$

The body fixed velocity twist of such a motion is thus,

$$
S_{B}=G^{-1} \frac{d}{d t} G=\nu\left(\begin{array}{cccc}
0 & -k_{1} & -k_{2} & 1 \\
k_{1} & 0 & 0 & 0 \\
k_{2} & 0 & 0 & 0 \\
0 & 0 & 0 & 0
\end{array}\right) .
$$

As a 6-dimensional twist vector this is,

$$
\mathbf{s}_{B}=\nu\left(\begin{array}{c}
0 \\
-k_{2} \\
k_{1} \\
1 \\
0 \\
0
\end{array}\right) .
$$

It is not difficult to see that this twist must lie in a IIB three-system of screws and since every screw in this system is a line the modulus is $p=0$.

The part of the proof showing that if the twist velocity lies in such a screw system then the motion must be a Bishop motion is precisely analogous to the case of the Frenet-Serret motions given above. 


\subsection{General Moving Frame Motion}

The Frenet-Serret and Bishop motions are particular types of motion based on moving frames to space curves. In general a moving frame for a curve is a frame with one axis aligned along the tangent to the curve. The other axes of the moving frame must be normal to the curve but are otherwise allowed to rotate freely about the curve. This prompts the question: When does a rigid-body motion move a frame in such a way that it is the moving frame to a regular curve? This is answered by the following theorem.

Theorem 2.3 A rigid-body motion moves a frame in such a way that it is the moving frame to a regular curve if and only if its body-fixed velocity lies in a 4-system of screws reciprocal to a IIA, $p=0$ 2-system. Again we must stipulate that the velocity in the translational direction is never zero.

Proof The trajectory of a point $\mathbf{p}_{0}$ in space is given by,

$$
\left(\begin{array}{c}
\mathbf{p}(t) \\
1
\end{array}\right)=G(t)\left(\begin{array}{c}
\mathbf{p}_{0} \\
1
\end{array}\right),
$$

where,

$$
G(t)=\left(\begin{array}{cc}
R(t) & \mathbf{q}(t) \\
0 & 1
\end{array}\right) .
$$

At some parameter value $t$ the tangent vector $\mathbf{t}$ to the trajectory will be given by the derivative of this,

$$
\left(\begin{array}{l}
\mathbf{t} \\
0
\end{array}\right)=\frac{d}{d t} G(t)\left(\begin{array}{c}
\mathbf{p}_{0} \\
1
\end{array}\right) .
$$

If $\mathbf{t}_{0}$ is the tangent vector to the curve at $t=0$, then the rigid motion will carry this to,

$$
G(t)\left(\begin{array}{c}
\mathbf{t}_{0} \\
0
\end{array}\right),
$$

at parameter value $t$. If the motion is generated as a moving frame of the trajectory then the tangent at $t$ and the image of $\mathbf{t}_{0}$ must agree, at least in 
direction,

$$
\frac{d}{d t} G(t)\left(\begin{array}{c}
\mathbf{p}_{0} \\
1
\end{array}\right)=\lambda(t) G(t)\left(\begin{array}{c}
\mathbf{t}_{0} \\
0
\end{array}\right)
$$

where $\lambda(t)$ is some scale factor. For regular curves the tangent will always exist so $\lambda(t)$ is never zero. Pre-multiplying by $G^{-1}$ gives,

$$
G^{-1} \frac{d}{d t} G\left(\begin{array}{c}
\mathbf{p}_{0} \\
1
\end{array}\right)=S_{B}\left(\begin{array}{c}
\mathbf{p}_{0} \\
1
\end{array}\right)=\lambda(t)\left(\begin{array}{c}
\mathbf{t}_{0} \\
0
\end{array}\right) .
$$

Here $S_{B}$ is the Lie algebra element representing the body-fixed velocity of the body. Writing this Lie algebra element as,

$$
S_{B}=\left(\begin{array}{cc}
\Omega_{B} & \mathbf{v}_{B} \\
0 & 0
\end{array}\right)
$$

we can extract the first three components of previous equation to get,

$$
\boldsymbol{\omega}_{B} \times \mathbf{p}_{0}+\mathbf{v}_{B}=\lambda(t) \mathbf{t}_{0} .
$$

Finally the scale factor $\lambda(t)$ is removed by taking the cross product with $\mathbf{t}_{0}$,

$$
\mathbf{t}_{0} \times\left(\boldsymbol{\omega}_{B} \times \mathbf{p}_{0}\right)+\mathbf{t}_{0} \times \mathbf{v}_{B}=\mathbf{0} .
$$

This is a system of three homogeneous linear equations for the six components of the body-fixed velocity twist $\mathbf{s}_{B}=\left(\boldsymbol{\omega}_{B}^{T}, \mathbf{v}_{B}^{T}\right)^{T}$. However, the three equations only have rank 2 so there are four possible solutions, or rather any solution is a linear combination of four linearly independent twists,

$$
\mathbf{s}_{B}=\alpha\left(\begin{array}{c}
\boldsymbol{\omega}_{1} \\
\mathbf{p}_{0} \times \boldsymbol{\omega}_{1}
\end{array}\right)+\beta\left(\begin{array}{c}
\boldsymbol{\omega}_{2} \\
\mathbf{p}_{0} \times \boldsymbol{\omega}_{2}
\end{array}\right)+\gamma\left(\begin{array}{c}
\boldsymbol{\omega}_{3} \\
\mathbf{p}_{0} \times \boldsymbol{\omega}_{3}
\end{array}\right)+\delta\left(\begin{array}{c}
\mathbf{0} \\
\mathbf{t}_{0}
\end{array}\right),
$$

where $\alpha, \beta, \gamma$ and $\delta$ are arbitrary constants. Another way of saying this is that $\mathbf{s}_{B}$ must lie in the screw system defined by the four linearly independent twists. This screw system is clearly of the required type, since the reciprocal system is clearly generated by the two linearly independent twists,

$$
\left(\begin{array}{c}
\mathbf{t}_{1} \\
\mathbf{0}
\end{array}\right) \text { and }\left(\begin{array}{c}
\mathbf{t}_{2} \\
\mathbf{0}
\end{array}\right)
$$


with $\mathbf{t}_{0} \cdot \mathbf{t}_{1}=0$ and $\mathbf{t}_{0} \cdot \mathbf{t}_{2}=0$.

Substituting this solution back into equation (5) it is clear that $\delta=\lambda(t)=\nu$. The speed of the motion $\nu$ is thus never zero.

On the other hand, if we were given a rigid-body motion whose body-fixed velocity twist lies in a 4 system of the given type then, as in the first two theorems above, we can change coordinates so that the screw system has the standard generators

$$
\left(\begin{array}{l}
\mathbf{i} \\
\mathbf{0}
\end{array}\right), \quad\left(\begin{array}{l}
\mathbf{j} \\
\mathbf{0}
\end{array}\right), \quad\left(\begin{array}{l}
\mathbf{k} \\
\mathbf{0}
\end{array}\right), \quad \text { and }\left(\begin{array}{l}
\mathbf{0} \\
\mathbf{i}
\end{array}\right) .
$$

Now suppose we take this new frame as the frame fixed in the moving body. From the computations above it is clear that the given motion will sweep the frame along the trajectory of the origin and the first axis of the moving frame will stay aligned with the tangent to this curve. Hence, the sequence of frames produced by the motion will form a moving frame field for the curve.

By thinking of rigid motions as curves in $S E(3)$ it is easy to see that parametrised motions can be multiplied together to produce new motions. The group of all such motions is essentially the loop group of $S E(3)$. A general moving frame motion can always be decomposed into the product a pair of motions as follows.

Theorem 2.4 Any moving frame motion on a regular curve $\gamma(t)$, can be factorised into the product of the regular Frenet-Serret motion generated by $\gamma(t)$ and a motion which rotates about the initial tangent of the curve $\gamma^{\prime}(0)$.

Proof Let $G(t)$ be an arbitrary moving frame motion. The theorem states that the motion must decompose into a product $G_{2}(t) G_{1}(t)$ where $G_{2}(t)$ is the regular Frenet-Serret motion and $G_{1}(t)$ is a rotational motion, that is

$$
G(t)=G_{2}(t) G_{1}(t)
$$


This can be rearranged to give $G_{2}(t)=G(t) G_{1}^{-1}(t)$, which can be solved as follows. Choose a coordinate frame in the moving body so that the velocity twist of the body for the motion $G(t)$ has the normal form,

$$
S_{B}=\nu\left(\begin{array}{cccc}
0 & -\lambda_{3} & \lambda_{2} & 1 \\
\lambda_{3} & 0 & -\lambda_{1} & 0 \\
-\lambda_{2} & \lambda_{1} & 0 & 0 \\
0 & 0 & 0 & 0
\end{array}\right) .
$$

In these coordinates the pure rotation will be of the form,

$$
G_{1}(t)=\left(\begin{array}{cccc}
1 & 0 & 0 & 0 \\
0 & \cos \theta & -\sin \theta & 0 \\
0 & \sin \theta & \cos \theta & 0 \\
0 & 0 & 0 & 1
\end{array}\right) .
$$

The velocity twist of the product can be computed as,

$$
G_{2}^{-1} \frac{d}{d t} G_{2}=G_{1} S_{B} G_{1}^{-1}-\frac{d \theta}{d t} X
$$

where $X$ is the Lie algebra element,

$$
X=\left(\begin{array}{cccc}
0 & 0 & 0 & 0 \\
0 & 0 & -1 & 0 \\
0 & 1 & 0 & 0 \\
0 & 0 & 0 & 0
\end{array}\right) \text {. }
$$

The action of $G_{1}$ on $S_{B}$ can be written in terms of six-vectors, so the above equation becomes,

$$
\mathbf{s}_{B 2}=\nu\left(\begin{array}{cccccc}
1 & 0 & 0 & 0 & 0 & 0 \\
0 & \cos \theta & -\sin \theta & 0 & 0 & 0 \\
0 & \sin \theta & \cos \theta & 0 & 0 & 0 \\
0 & 0 & 0 & 1 & 0 & 0 \\
0 & 0 & 0 & 0 & \cos \theta & -\sin \theta \\
0 & 0 & 0 & 0 & \sin \theta & \cos \theta
\end{array}\right)\left(\begin{array}{c}
\lambda_{1} \\
\lambda_{2} \\
\lambda_{3} \\
1 \\
0 \\
0
\end{array}\right)-\frac{d \theta}{d t}\left(\begin{array}{c}
1 \\
0 \\
0 \\
0 \\
0 \\
0
\end{array}\right) .
$$


Now by setting $\theta=\arctan \left(\lambda_{2} / \lambda_{3}\right)$ it can be seen that the velocity twist for the motion $G_{2}$ lies in a $I B_{0} 3$-system with $p_{a}=p_{b}=0$, hence by theorem 2.1 above is a regular Frenet-Serret motion. It is clear from the form of $G_{1}$ that both $G$ and $G_{2}$ are generated by the same curve: the trajectory of the origin in the moving coordinate frame.

Conversely, it is easy to see that the product of a regular Frenet-Serret motion with a rotation about the initial tangent produces a general moving frame motion.

Notice that a Bishop motion is a particular type of moving frame motion and so can be produced in this way. In particular the angular function $\theta$ must be chosen so that $\lambda_{1}$ vanishes, that is $\theta=\int \nu \tau d t+\theta_{0}$. The different Bishop motions mentioned above correspond to the choice of integration constant $\theta_{0}$.

This type of general moving frame motion seems to occur in many applications, in particular it is the possible motion of an aeroplane which can roll, pitch and yaw but must maintain a positive forward speed. It can also be used to model the motions of a roller coaster, a neutrally buoyant submarine, a car without suspension or to model directional drilling of oil-wells using steerable motors. These ideas have been applied in Robot vision by Duric et al ${ }^{11}$ for example.

\section{$3 \quad$ Needle Steering}

Recently in the literature there have been several works addressing the problem of needle steering. The needle in question is long and flexible with a bevelled tip and is used as an active cannula in surgical applications. When the needle is inserted into soft tissue its path curves due to the asymmetrical force generated by the bevelled tip. 


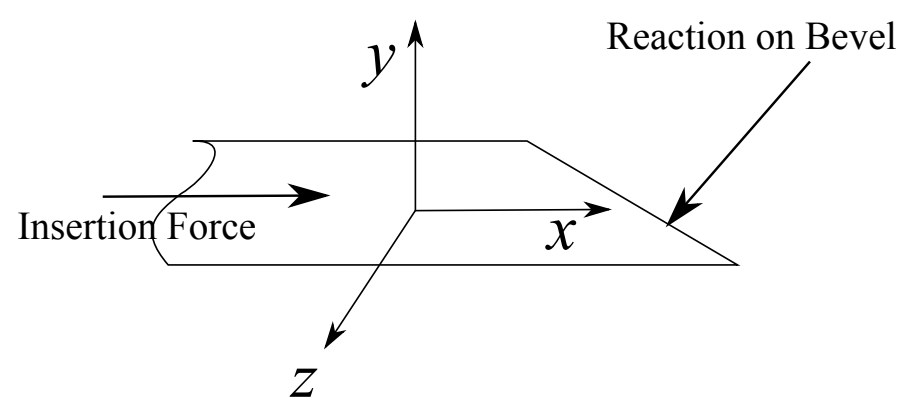

Figure 1: Body-fixed coordinate frame for a needle tip

In the following some of the ideas introduced above are used to study an idealised model of this physical device.

\subsection{Kinematic equations}

Park et $a l^{13}$ derive a kinematic model of the needle by considering an equivalent unicycle. This model was modified later by Webster et $a l^{12}$ who considered an equivalent bicycle. The derivation of the model here is given without reference to an equivalent vehicle.

The needle has two controls, the speed of insertion can be controlled and the rate at which the needle is turned about its axis can be regulated. Suppose the needle is inserted at a rate of $\nu$ units per second (in practice a realistic insertion rate will be a small fraction of one meter per second), the effect of insertion will be a force along the axis of the needle but there will also be a reaction force on the bevel caused by the surrounding tissue and possibly a frictional force too. All these forces lie in a plane and hence the effects of the all the forces can be regarded as a force plus a torque about a line normal to the plane. The torque will bend the needle and cause the tip of the needle to rotate. It is convenient to take the axis that the tip turns about as the $z$-axis in the tip, see figure 1 . 
Inserting the needle at a rate $\nu$ will produce a twist on the tip given by,

$$
\nu \mathbf{s}_{i}=\nu\left(\begin{array}{c}
0 \\
0 \\
0 \\
1 \\
0 \\
0
\end{array}\right)+\nu \kappa\left(\begin{array}{l}
0 \\
0 \\
1 \\
0 \\
0 \\
0
\end{array}\right)=\nu\left(\begin{array}{l}
0 \\
0 \\
\kappa \\
1 \\
0 \\
0
\end{array}\right),
$$

where $\kappa$ is some proportionality constant. This constant will depend on the details of the interaction between the bevel and the surrounding tissue.

This very simple model assumes that the torsional stiffness of the needle is very high, so that any twist of the needle is instantaneously transmitted to the needle tip. Turning the needle at a rate of $\mu$ radians per second will result in a twist on the tip given by,

$$
\mu \mathbf{s}_{t}=\mu\left(\begin{array}{c}
1 \\
0 \\
0 \\
0 \\
0 \\
0
\end{array}\right)
$$

The total twist in the body-fixed frame will be,

$$
\mathbf{s}_{B}=\nu \mathbf{s}_{i}+\mu \mathbf{s}_{t} .
$$

From the results of section 2.2 this is a Frenet-Serret motion. However, in this case the curvature $\kappa$, is constant. Moreover, it is easy to see that $\nu$, the insertion rate, is equal to the speed of the generating curve for the motion and the torsion of the generating curve is given by $\tau=\mu / \nu$.

In this case the velocity twist of the motion $\mathbf{s}_{B}$, clearly lies in a 2 -system of screws, generated by $\mathbf{s}_{i}$ and $\mathbf{s}_{t}$. Clearly, such a 2 -system cannot contain any infinite pitch screws, so it is a type $I$ system. The pitch of a general screw in 
the system is $\mu \nu /\left(\mu^{2}+\nu^{2} \kappa^{2}\right)=\tau /\left(\tau^{2}+\kappa^{2}\right)$, which is not a constant and so this is a $I A 2$-system. Such 2-systems are classified by a pair of moduli $p_{a}, p_{b}$ also known as the principal pitches of the system. The principal screws of the system are the screws in the system satisfying,

$$
\mathbf{s}_{a}^{T} Q_{\infty} \mathbf{s}_{b}=0, \quad \mathbf{s}_{a}^{T} Q_{0} \mathbf{s}_{b}=0
$$

In this case we have,

$$
\mathbf{s}_{a}=\kappa \mathbf{s}_{i}+\mathbf{s}_{t}, \quad \mathbf{s}_{b}=-\kappa \mathbf{s}_{i}+\mathbf{s}_{t} .
$$

The principal pitches are the pitches of these principal screws, here it is easy to compute the principal pitches are $p_{a}=1 /(2 \kappa)$ and $p_{b}=-1 /(2 \kappa)$. See the appendix for more details.

In summary, for this simple model, the motion of the needle tip is characterised as a motion in which the body-fixed twist velocity remains in a $I A$ 2 -system with principal pitches $\pm 1 /(2 \kappa)$.

\subsection{Unicycle and Bicycle Models}

Experimental and simulation results reported by Alterovitz et $a l^{14}$ suggest that the curves followed by these needles are curves with constant curvature at least when there is no deformation of the surrounding tissue. Later, this assumption was taken as a starting point for modelling by Duindam et al,.$^{4}$

The model given in the previous section is the same as both the models given by Park et al and Webster et al. The rigid-body motion of the needle tip will be the same in both models. The only difference between the models is relation between the point chosen to represent the tip of the needle and the origin of the coordinate frame. In the unicycle model the origin of coordinates is located at the middle of the needle tip. Hence in this model the curve described by the needle will be the curve of constant curvature which generates the motion. 
For the bicycle model the origin of the coordinate system is displaced slightly from the needle tip. Hence in this model the curve followed by the needle tip is slightly different. However, it must be emphasised that in both models the rigidbody motion that the needle tip performs is a Frenet-Serret motion generated by a curve of constant curvature.

In the bicycle model presented by Webster et al the origin of the coordinate frame traces a constant curvature trajectory and the tip of the needle is located a small distance along the tangent vector to the curve. Hence the curve traced by the needle will be slightly different from a constant curvature trajectory. It is possible to say a little about these curves. In particular the curvature and torsion of the curves can be estimated.

Choose a global fixed coordinate frame which coincides with the body-fixed frame when $t=0$. In these coordinates the end-point of the needle at $t=0$ will be given by,

$$
\mathbf{r}_{0}=\left(\begin{array}{l}
x \\
0 \\
0 \\
1
\end{array}\right)
$$

Note that in ref. ${ }^{12}$ the distance $x$ is denoted $\ell_{2}$ and the coordinates are oriented so that the $z$-axis lies along the tangent to the constant curvature trajectory.

Now subsequent positions of the needle tip will be given by,

$$
\mathbf{r}(t)=G(t) \mathbf{r}_{0} .
$$

The first derivative of this gives the velocity of the needle tip and the tangent to the curve,

$$
\frac{d}{d t} \mathbf{r}=G S_{B} \mathbf{r}_{0}
$$

where $S_{B}$ is the $4 \times 4$ matrix representing the twist velocity of the motion as given in equation (3) above. Note that the curvature $\kappa$ will be taken as a 
constant in the following. The speed of the needle-tip can be easily computed from the magnitude of this vector to be, $\nu_{1}=\nu \sqrt{1+x^{2} \kappa^{2}}$. Notice that for the unicycle model the speed of the curve is the same as the insertion rate of the needle $\nu$.

The acceleration of the needle-tip is given by,

$$
\frac{d^{2}}{d t^{2}} \mathbf{r}=G\left(S_{B}^{2}+\dot{S}_{B}\right) \mathbf{r}_{0}
$$

Using standard formulas to compute the curvature of the curve we have,

$$
\kappa_{1}=\frac{\nu \kappa}{\nu_{1}^{3}} \sqrt{a_{4} x^{4}+a_{3} x^{3}+a_{2} x^{2}+a_{1} x+a_{0}},
$$

where,

$$
\begin{aligned}
& a_{4}=\nu^{6} \kappa^{4}\left(\kappa^{2}+\tau^{2}\right), \\
& a_{3}=-2 \nu^{4} \kappa^{4}(\nu+\nu \dot{\nu}-\dot{\nu}), \\
& a_{2}=\nu^{2} \kappa^{2}\left(\nu^{4}\left(2 \kappa^{2}+\tau^{2}\right)+(\nu+\nu \dot{\nu}-\dot{\nu})^{2}\right), \\
& a_{1}=-2 \nu^{4} \kappa^{2}(\nu+\nu \dot{\nu}-\dot{\nu}), \\
& a_{0}=\nu^{6} \kappa^{2} .
\end{aligned}
$$

Assuming that $x$ is small, it is probably more useful to write this as a Taylor expansion,

$$
\kappa_{1}=\kappa+x \frac{\kappa}{\nu^{2}}(\nu+\nu \dot{\nu}-\dot{\nu})+x^{2} \frac{\kappa}{2}\left(\tau^{2}-\kappa^{2}\right)+O\left(x^{3}\right) .
$$

To compute the torsion requires the third derivative,

$$
\frac{d^{3}}{d t^{3}} \mathbf{r}=G\left(S_{B}^{3}+2 S_{B} \dot{S}_{B}+\dot{S}_{B} S_{B}+\ddot{S}_{B}\right) \mathbf{r}_{0}
$$

Again using standard formulas the torsion of the curve is given as,

$$
\tau_{1}=\frac{b_{3} x^{3}+b_{2} x^{2}+b_{1} x+b_{0}}{c_{4} x^{4}+c_{3} x^{3}+c_{2} x^{2}+c_{1} x+c_{0}}
$$


where,

$$
\begin{aligned}
b_{3} & =\nu^{3} \kappa^{2} \dot{\tau} \\
b_{2} & =\nu^{4}\left(\kappa^{2}+\tau^{2}\right) \tau-\nu \dot{\tau}(\nu+\nu \dot{\nu}-\dot{\nu})-3 \dot{\nu} \tau(\nu+\nu \dot{\nu}-\dot{\nu})+\nu \tau(2 \dot{\nu}+\nu \ddot{\nu}-\ddot{\nu}), \\
b_{1} & =-3 \nu^{2} \tau(\nu+\nu \dot{\nu}-\dot{\nu})+\nu^{3} \dot{\tau} \\
b_{0} & =\nu^{4} \tau .
\end{aligned}
$$

and where,

$$
\begin{aligned}
& c_{4}=\nu^{4} \kappa^{2}\left(\kappa^{2}+\tau^{2}\right), \\
& c_{3}=-2 \nu^{2} \kappa^{2}(\nu+\nu \dot{\nu}-\dot{\nu}, \\
& c_{2}=\nu^{4}\left(2 \kappa^{2}+\tau^{2}\right)+(\nu+\nu \dot{\nu}-\dot{\nu})^{2}, \\
& c_{1}=-2 \nu^{2}(\nu+\nu \dot{\nu}-\dot{\nu}), \\
& c_{0}=\nu^{4} .
\end{aligned}
$$

For small $x$ the Taylor expansion of this is,

$$
\begin{aligned}
\tau_{1}= & \tau+x \frac{\tau(\nu+\nu \dot{\nu}-\dot{\nu})+\nu \dot{\tau}}{\nu^{2}}+ \\
& x^{2} \frac{\tau\left(\nu^{3} \kappa^{2}-3(1+\dot{\nu})(\nu+\nu \dot{\nu}-\dot{\nu})+(2 \dot{\nu}+\nu \ddot{\nu}-\ddot{\nu})\right)-\dot{\tau}((\nu+\nu \dot{\nu}-\dot{\nu})}{\nu^{3}}+O\left(x^{3}\right) .
\end{aligned}
$$

These formulas may be useful when trying to identify the parameter $x$ from experimental data. Notice that if we assume that the insertion rate $\nu$ is constant then the curvature of the needle's path is constant to first order in $x$.

\subsection{Curves with constant curvature}

From the above it is clear that curves with constant curvature are important for the needle steering problem. However, very few examples of curves with constant curvature seem to be known. Of course one could decide on a torsion function and then solve the Frenet-Serret equations numerically for a fixed curvature. The results however, would not be very useful for path planning. 
The circular helix is a curve with both constant curvature and torsion. Koch and Englehardt ${ }^{15}$ give a method for interpolating curves from pieces of circular helix. These curves will have constant curvature but the torsion will be discontinuous at the junctions of different pieces. This might be achieved by stopping the insertion of the needle at a junction point, reorienting the tip and then continuing the insertion. A better solution might be to use curves with smoothly varying torsion.

In 1909 Salkowski ${ }^{16}$ introduced a family of curves with constant curvature but smoothly varying torsion, see also more recent work by Monterde. ${ }^{17}$ These curves are given by the following parametrisation:

$$
\begin{aligned}
& x(t)=\frac{-1}{\sqrt{1+m^{2}}}\left(\frac{1-n}{4(1+2 n)} \sin (1+2 n) t+\frac{1+n}{4(1-2 n)} \sin (1-2 n) t+\frac{1}{2} \sin t\right), \\
& y(t)=\frac{1}{\sqrt{1+m^{2}}}\left(\frac{1-n}{4(1+2 n)} \cos (1+2 n) t+\frac{1+n}{4(1-2 n)} \cos (1-2 n) t+\frac{1}{2} \cos t\right), \\
& z(t)=\frac{1}{4 m \sqrt{1+m^{2}}} \cos 2 n t,
\end{aligned}
$$

where $n=m / \sqrt{1+m^{2}}$.

The speed, curvature and torsion of these curves are given by,

$$
\nu=\frac{\cos n t}{\sqrt{1+m^{2}}}, \quad \kappa=1, \quad \tau=-\tan n t
$$

Notice that for needle steering it would necessary to use the portion of the curve between the cusps at $t= \pm \pi /(2 n)= \pm \pi \sqrt{1+m^{2}} /(2 m)$. In fact it may be useful to begin and end the needle motion at or near a cusp since at these point the velocity of the motion is zero. Hence the insertion will begin with the needle at rest and end with the needle gradually slowing to rest. Unfortunately there are some practical problems such a scheme, the distance between the cusp points and the direction of the tangent to the curve at these points is rather tightly constrained by the parameter $m$. This means that only a small range of 

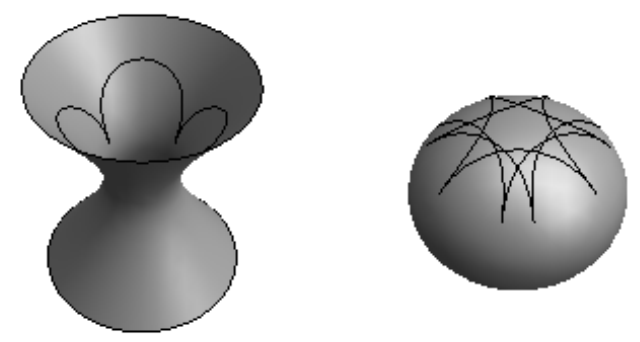

Figure 2: Salkowski curves lying on quadrics. The left one has $m=0.42$ and the right has $m=1.0$.

possible start and end points can be specified. (Technically the tangent to the curve at a cusp is undefined, however, it is possible to find the initial direction of the curve segment under consideration).

For the problem of planning a path for a flexible needle it is probably useful to know about surfaces that the curves lie on. For example, if the tissue contains physiological structures that it is important to avoid then it could be surrounded by a virtual surface on which the path of the needle will lie. The helices mentioned above, lie on circular cylinders the Salkowski curves lie on circular ellipsoids or hyperboloids. It is possible to show that the equation of the quadric that these curves lie on is given by

$$
A\left(x^{2}+y^{2}\right)-B-C(z+D)^{2}=0,
$$

where

$$
\begin{aligned}
& A=1+m^{2} \\
& B=27 n^{4} /\left(4\left(1-n^{2}\right)\left(1-4 n^{2}\right)^{2}\right) \\
& C=4 n^{2} /\left(\left(1-n^{2}\right)\left(1-4 n^{2}\right)\right) \\
& D=\left(1+2 n^{2}\right) /(4 n)
\end{aligned}
$$

The type of quadric that this equation represents depend on the sign of $C$, 
this in turn is dependant on the value of $m$. If $m<1 / \sqrt{3}$ then $C>0$ and the quadric is a hyperboloid of one sheet. When $m>1 / \sqrt{3}, C<0$ and the quadric is an ellipsoid. Both of these quadrics will be circularly symmetric about the $z$-axis, see figure 2 .

The above discussion on curves of constant curvature is only intended to set forth some preliminary ideas. Clearly, a better knowledge of such curves would be helpful.

\section{Conclusion}

The characterisations of the Frenet-Serret and Bishop motions in terms of properties of their velocity twists is believed to be novel. This allows one to check easily whether or not any proposed motion is of one of these types.

Much previous work in this area has tended to concentrate on the trajectories of points in space produced by rigid-body motions. Here the view is taken that the primary object of interest is the curve in the group which defines the motion. Though the rigid motions discussed in this work are all based on curves in space, it is hoped that the advantages of this viewpoint are demonstrated by the work presented here. The advantage is perhaps most easily seen when discussing motions defined in other ways. For example many special rigidbody motions are defined as solutions to variational problems, for example the stationary acceleration motions, ${ }^{7}$ stationary jerk motions and even the dynamics of rigid bodies. These motions also have applications in computer animation and robotics.

From the results presented here it can be seen that many types of motion can be characterised in a similar fashion. A one-parameter finite screw motion could be characterised as a motion whose twist velocity, in either a globally fixed frame or a frame fixed in the body, is constant - a 1-system of screws. Spherical 
motions, that is motions with a fixed point could be characterised as motions whose twist velocities lie in a $\operatorname{IIA}(p=0) 3$-system. Planar motions, motions in a fixed plane must have velocity twists lying in a $I I C(p=0) 3$-system. Pure translations could be characterised as motions whose velocity twist lies in the IID 3-system.

New types of special motions could also be defined by requiring that the body-fixed velocity twist remains in some other screw system. Restricting the body-fixed velocity to a $I A_{1}$ system for example, would produce interesting motions not related to any moving frame field on any space curve.

It is also clear that these sorts of motions are important for modelling vehicles with actuators which provide forces and torques lying on constant screws relative to the vehicle itself.

The results for needle steering are only intended to be indicative. The models used to represent the motion of the needle are very simple and probably not accurate enough for real tissue. However, the work does seem to suggest that it would be useful to know more about curves of constant curvature lying on simple surfaces, a neglected subject in classical differential geometry.

\section{Acknowledgments}

This work was has been much improved following the comment of the anonymous reviewers.

\section{References}

[1] F. Klok, 1986, "Two moving coordinate frames for sweeping along a 3D trajectory", Computer Aided Geometric Design, 3:217-229. 
[2] M. Wagner and B. Ravani, 1997, "Curves with rational Frenet-Serret motion", Computer Aided Geometric Design, 15:79-101.

[3] O. Bottema and B. Roth, 1990, Theoretical Kinematics, Dover Publications, New York.

[4] V. Duindam, J. Xu, R. Alterovitz, S. Sastry and K. Goldberg, 2010 "Threedimensional motion planning algorithms for steerable needles using inverse kinematics", International Journal of Robotics Research, 29(7):789-800.

[5] R.L. Bishop, 1975, "There is more than one way to frame a curve", Am. Math. Monthly, 82:246-251.

[6] R. Revani and A. Meghdari, 2004, "Spatial motions based on rational Frenet-Serret curves", IEEE International Conference on Systems, Man and Cybernetics, 5:4456-4461.

[7] J.M. Selig, 2007, "Curves of stationary acceleration in $\mathrm{SE}(3)$ ", IMA J Math Control Info., 24(1):95-113.

[8] R. T. Farouki, C. Giannelli and A. Sestini, 2009 "Helical polynomial curves and double Pythagorean hodographs I. Quaternion and Hopf map representations", Journal of Symbolic Computation, 44: 161-179.

[9] C.G. Gibson and K.H. Hunt, 1990, "Geometry of screw systems", Mechanism and Machine Theory, 25:1-27.

[10] P.S. Donelan and C.G. Gibson, 1993, "On the hierarchy of screw systems", Acta Appl. Math., 32:267-296.

[11] Z. Duric, A. Rosenfeld and L. S. Davis, 1995, "Egomotion analysis based on the Frenet-Serret motion model", International Journal of Computer Vision, 15:703-712. 
[12] R. J. Webster III, J. S. Kim, N. J. Cowan, G. S. Chirikjian and A. M. Okamura, 2006, "Nonholonomic Modeling of Needle Steering", The International Journal of Robotics Research, 25(5-6):509-525.

[13] W. Park, J. S. Kim, Y. Zhou, N. J. Cowan, A. M. Okamura, G.S. Chirikjian, 2005, "Diffusion-based motion planning for a nonholonomic flexible needle model", IEEE International Conference on Robotics and Automation (ICRA), Barcelona, Spain.

[14] R. Alterovitz, K. Goldberg and A. Okamura, 2005, "Planning for steerable bevel-tip needle insertion through 2D soft tissue with obstacles", Proceedings of the 2005 IEEE International Robotics and Automation, pp. 16401645.

[15] R. Koch and C. Englehardt, 1998, "Closed Space Curves of Constant Curvature Consisting of Arcs of Circular Helices", Journal for Geometry and Graphics,2(1):17-31.

[16] E. Salkowski, 1909, "Zur Transformation von Raumkurven", Mathematische Annalen, 66(4):517-557.

[17] J. Monterde, 2009, "Salkowski curves revisited: A family of curves with constant curvature and non-constant torsion", Computer Aided Geometric Design, 26(3):271-278.

[18] R.S. Ball. The Theory of Screws, Cambridge University Press, Cambridge, 1900.

\section{Appendix - Introduction to Screw Theory}

Screw theory was introduced by R.S. Ball in the late 1800s. Ball's work culminated in the publication of his "treatise", ${ }^{18}$ in 1900 . For Ball a screw was a 
geometric figure, a line in space together with a real number called the pitch of the screw. Giving an "amplitude" for a screw turns it into, either a twist - an infinitesimal rigid-body motion, or a wrench which is the combination of a force with a moment.

A modern approach identifies twists with elements of the Lie algebra of the group of rigid-body displacements, $S E(3)$. A screw is then technically a ray through the origin of the Lie algebra. In other word an element of the projective space formed from the Lie algebra.

By Charles's theorem a general rigid -body displacement is a finite screw motion, a rotation about a line in space followed by a translation in the direction of the line. In the limit as the angle of rotation becomes small the general finite screw motion becomes an infinitesimal screw motion. The Lie algebra of $S E(3)$ is six-dimensional, hence a twist can be written as a six-dimensional vector. It is often convenient to partition a twist into a pair of 3 -vectors,

$$
\mathrm{s}=\left(\begin{array}{l}
\boldsymbol{\omega} \\
\mathbf{v}
\end{array}\right)
$$

here $\boldsymbol{\omega}$ is a vector in the direction of the infinitesimal screw motion's axis. The second 3-vector $\mathbf{v}$ can be decomposed as,

$$
\mathbf{v}=p \boldsymbol{\omega}+\mathbf{r} \times \boldsymbol{\omega}
$$

where $\mathbf{r}$ is the position vector of any point on the axis of the motion, so that the vector product, $\mathbf{r} \times \boldsymbol{\omega}$, is the moment of the axis. The quantity $p$ is the pitch of the motion and gives the amount of translational motion for each radian of rotational motion. A pure rotation is given by a twist with pitch $p=0$. In a pure translational motion $\boldsymbol{\omega}=\mathbf{0}$ and in these circumstances the pitch is usually said to be infinite although technically it is undefined.

The group $S E(3)$ acts on its own Lie algebra by the adjoint representation. The adjoint representation can be written in terms of $6 \times 6$ matrices, partitioned 
so that,

$$
\left(\begin{array}{l}
\boldsymbol{\omega} \\
\mathbf{v}
\end{array}\right) \longmapsto\left(\begin{array}{cc}
R & 0 \\
T R & R
\end{array}\right)\left(\begin{array}{l}
\boldsymbol{\omega} \\
\mathbf{v}
\end{array}\right)
$$

Where $R$ is the $3 \times 3$ rotation matrix of the displacements and $T$ is the $3 \times 3$ antisymmetric matrix corresponding to the translation vector $\mathbf{t}$ of the displacement.

There are two quadratic forms defined on the twists which are invariant with respect to the adjoint representation. They are the reciprocal or Klein form, given by:

$$
\mathbf{s}^{T} Q_{0} \mathbf{s}=\left(\boldsymbol{\omega}^{T}, \mathbf{v}^{T}\right)\left(\begin{array}{cc}
0 & I_{3} \\
I_{3} & 0
\end{array}\right)\left(\begin{array}{l}
\boldsymbol{\omega} \\
\mathbf{v}
\end{array}\right)=2 \boldsymbol{\omega} \cdot \mathbf{v},
$$

and the Killing form,

$$
\mathbf{s}^{T} Q_{\infty} \mathbf{s}=\left(\boldsymbol{\omega}^{T}, \mathbf{v}^{T}\right)\left(\begin{array}{cc}
I_{3} & 0 \\
0 & 0
\end{array}\right)\left(\begin{array}{l}
\boldsymbol{\omega} \\
\mathbf{v}
\end{array}\right)=\boldsymbol{\omega} \cdot \boldsymbol{\omega},
$$

where $I_{3}$ is the $3 \times 3$ identity matrix. In terms of these invariants the pitch of the twist is given by,

$$
p=\frac{\mathbf{s}^{T} Q_{0} \mathbf{s}}{2 \mathbf{s}^{T} Q_{\infty} \mathbf{s}}
$$

A screw system is a linear system of screws, in terms of twists we can write a 2-system of screws as,

$$
\mathcal{S}=\left\{\mathbf{s}: \mathbf{s}=a \mathbf{s}_{1}+b \mathbf{s}_{2}\right\} .
$$

The twists $\mathbf{s}_{1}$ and $\mathbf{s}_{2}$ are called the basis of the screw system. The coefficients $a$ and $b$ are real numbers. Three, four and five systems are defined similarly using three, four and five twists respectively.

There are two groups which act on these screw systems, first the group of rigid-body displacements $S E(3)$ acts on any screw-system via the adjoint representation of the group on the basis screws. The second group action on a screw-system is the action of the general linear group which acts by a change of basis. For the two-system above a change of basis would be,

$$
\begin{aligned}
& \mathbf{s}_{1}^{\prime}=a \mathbf{s}_{1}+b \mathbf{s}_{2} \\
& \mathbf{s}_{2}^{\prime}=c \mathbf{s}_{1}+d \mathbf{s}_{2}
\end{aligned}
$$


with $\operatorname{det}\left(\begin{array}{ll}a & b \\ c & d\end{array}\right) \neq 0$. Two screw-systems are considered to be the same if they only differ by a change of basis. In many cases we want to consider two screw-systems to be equivalent if they differ by a rigid displacement.

Fortunately there is a classification of screw-systems up-to equivalence under the action of these two groups. This classification was first studied by Hunt and then a formal classification and proof was given by Gibson and Hunt. ${ }^{9}$ A later work by Donelan and Gibson ${ }^{10}$ gives further details.

The Gibson-Hunt classification can be outlined as follows. First of all we only need to consider 1,2 and 3-systems of screws; the 4 and 5 -systems are classified by their reciprocal 2 and 1-systems respectively. The reciprocal of a 4 -system for example is given by,

$$
\overline{\mathcal{S}}=\left\{\mathbf{s}: \mathbf{s}^{T} Q_{0}\left(a \mathbf{s}_{1}+b \mathbf{s}_{2}+c \mathbf{s}_{3}+d \mathbf{s}_{4}\right)=0\right\},
$$

for all $a, b, c$ and $d$.

Next the 1-systems are completely classified by their pitch. That is, two twists differ by a rigid-body displacement if and only if their pitches are equal.

To classify 2 and 3 systems (and hence 4 and 5 -systems), consider the family of quadric hypersurfaces in $\mathbb{P}^{5}$ determined by the $6 \times 6$ symmetric matrices,

$$
Q_{p}=\alpha Q_{0}+\beta Q_{\infty}
$$

where $Q_{0}$ and $Q_{\infty}$ are as described above. These quadrics are known as the pitch quadrics. In this geometric view a screw-system is a linear subspace of $\mathbb{P}^{5}$, that is a 2 -system corresponds to a line, a 3 -system to a plane. Now it might happen that the screw-system under consideration lies entirely in one of the pitch quadrics, this would be referred to as a II system of screws. Notice that in a II system (almost) all the screws will have the same pitch. If the screws in a screw system have different pitches then the system is called a $I$ system and it will intersect (almost) all the pitch quadrics. This partitions the screw- 
systems into two possible classes. They can be further sub-divided by observing how they meet the pitch-quadric $Q_{\infty}$, that is $Q_{p}$ with $\alpha=0$. This quadric is degenerate and forms a 2-plane which lies in all of the other pitch quadrics. Now the linear space corresponding to the screw-system under consideration might not meet $Q_{\infty}$, in this case we say that the system is an $A$ system. If the screw-system meets $Q_{\infty}$ in a point then we have a $B$ system and so forth.

So we can find 2-systems of class $I A, I B, I I A, I I B$ and IIC. The 3 -systems fall into classes, IA, IB, IC, IIA, IIB, IIC and IID. The IID system is the unique 3 -system that consists of all of $Q_{\infty}$, that is the set of all infinite pitch twists. In general each of these classes contains many screw-systems and these can be classified more finely. The intersection of the screw-systems with the pitch quadrics is a much studied object in classical Algebraic geometry - a pencil of quadrics. The final level of classification distinguishes screw-systems for which the projective type of this pencil of quadrics is different. In many cases there are continuous families of equivalent screw-systems and these will be distinguished by one or more moduli. The simplest example here is the 1-systems, there is a one-parameter family of equivalence classes of 1-systems these are distinguished by a single modulus - the pitch.

As an example consider the following screw system from the text above,

$$
\mathbf{s}_{B}=\nu\left(\begin{array}{c}
\tau \\
0 \\
\kappa \\
1 \\
0 \\
0
\end{array}\right) .
$$

That is,

$$
\mathcal{S}=\left\{\mathbf{s}: \mathbf{s}=\alpha \mathbf{s}_{1}+\beta \mathbf{s}_{3}+\gamma \mathbf{s}_{4}\right\}
$$

where $\alpha, \beta$ and $\gamma$ are arbitrary coefficients, and $\mathbf{s}_{1}, \mathbf{s}_{3}, \mathbf{s}_{4}$ are as given above in 
section 2.2

This is clearly a 3 -system since the three basis screws $\mathbf{s}_{1}, \mathbf{s}_{3}$ and $\mathbf{s}_{4}$ are linearly independent. The pitch of $\mathbf{s}$ is clearly $\alpha \gamma /\left(\alpha^{2}+\beta^{2}\right)$. This is not in general constant, hence in terms of the Gibson-Hunt classification, this must be a type $I$ system. Moreover, it is clear that the system contains a single screw of infinite pitch, $\mathbf{s}_{4}$, so this is a $I B$ 3-system.

There are two finer classes of these 3-systems; the $I B_{0}$ systems and the $I B_{3}$ systems. These are distinguished by the projective type of the pencil of conics formed by the pitch quadrics, more specifically here, by the type of degenerate quadrics in the pencil. In general a pencil of conics will contain 3 degenerate conics, where a degenerate conic is a line-pair. According to Donelan and Gibson, ${ }^{10}$ the pencil corresponding to a $I B_{0}$ system contains a single real line-pair and a repeated complex line-pair. The pencil corresponding to the $I B_{3}$ systems is a singular pencil with two distinct repeated lines.

A general $I B_{0}$ system has basis screws given by the normal form,

$$
\mathbf{s}_{a}=\left(\begin{array}{c}
1 \\
0 \\
0 \\
p_{a} \\
0 \\
0
\end{array}\right), \quad \mathbf{s}_{b}=\left(\begin{array}{c}
0 \\
1 \\
0 \\
0 \\
p_{a} \\
0
\end{array}\right), \quad \mathbf{s}_{c}=\left(\begin{array}{c}
0 \\
0 \\
0 \\
1 \\
0 \\
p_{b}
\end{array}\right) .
$$

The numbers $p_{a}$ and $p_{b}$ are known as moduli. These give the finest level of classification, Donelan and Gibson show that two $I B_{0}$ systems with moduli $p_{a}, p_{b}$ and $q_{a}, q_{b}$ can be rigidly-transformed into each other if and only if $p_{a}=q_{a}$ and $p_{b}^{2}=q_{b}^{2}$. 
The pitch quadrics for the normal form are given by,

$$
\begin{array}{r}
(\alpha, \beta, \gamma)\left(\begin{array}{ccc}
\mathbf{s}_{\mathbf{a}}{ }^{T} Q_{0} \mathbf{s}_{a} & \mathbf{s}_{\mathbf{a}}{ }^{T} Q_{0} \mathbf{s}_{b} & \mathbf{s}_{\mathbf{a}}{ }^{T} Q_{0} \mathbf{s}_{c} \\
\mathbf{s}_{\mathbf{b}}^{T} Q_{0} \mathbf{s}_{a} & \mathbf{s}_{\mathbf{b}}{ }^{T} Q_{0} \mathbf{s}_{b} & \mathbf{s}_{\mathbf{b}}{ }^{T} Q_{0} \mathbf{s}_{c} \\
\mathbf{s}_{\mathbf{c}}^{T} Q_{0} \mathbf{s}_{a} & \mathbf{s}_{\mathbf{c}}{ }^{T} Q_{0} \mathbf{s}_{b} & \mathbf{s}_{\mathbf{c}}{ }^{T} Q_{0} \mathbf{s}_{c}
\end{array}\right)\left(\begin{array}{l}
\alpha \\
\beta \\
\gamma
\end{array}\right)= \\
(\alpha, \beta, \gamma)\left(\begin{array}{ccc}
2 p_{a} & 0 & 1 \\
0 & 2 p_{a} & 0 \\
1 & 0 & 0
\end{array}\right)\left(\begin{array}{l}
\alpha \\
\beta \\
\gamma
\end{array}\right)=0,
\end{array}
$$

and

$$
\begin{aligned}
(\alpha, \beta, \gamma)\left(\begin{array}{ccc}
\mathbf{s}_{\mathbf{a}}{ }^{T} Q_{\infty} \mathbf{s}_{a} & \mathbf{s}_{\mathbf{a}}{ }^{T} Q_{\infty} \mathbf{s}_{b} & \mathbf{s}_{\mathbf{a}}{ }^{T} Q_{\infty} \mathbf{s}_{c} \\
\mathbf{s}_{\mathbf{b}}^{T} Q_{\infty} \mathbf{s}_{a} & \mathbf{s}_{\mathbf{b}}{ }^{T} Q_{\infty} \mathbf{s}_{b} & \mathbf{s}_{\mathbf{b}}{ }^{T} Q_{\infty} \mathbf{s}_{c} \\
\mathbf{s}_{\mathbf{c}}{ }^{T} Q_{\infty} \mathbf{s}_{a} & \mathbf{s}_{\mathbf{c}}{ }^{T} Q_{\infty} \mathbf{s}_{b} & \mathbf{s}_{\mathbf{c}}{ }^{T} Q_{\infty} \mathbf{s}_{c}
\end{array}\right)\left(\begin{array}{l}
\alpha \\
\beta \\
\gamma
\end{array}\right)= \\
(\alpha, \beta, \gamma)\left(\begin{array}{lll}
1 & 0 & 0 \\
0 & 1 & 0 \\
0 & 0 & 0
\end{array}\right)\left(\begin{array}{l}
\alpha \\
\beta \\
\gamma
\end{array}\right)=0 .
\end{aligned}
$$

The degenerate conics in the pencil are found by solving the characteristic equation,

$$
\operatorname{det}\left(\lambda\left(\begin{array}{ccc}
2 p_{a} & 0 & 1 \\
0 & 2 p_{a} & 0 \\
1 & 0 & 0
\end{array}\right)+\mu\left(\begin{array}{lll}
1 & 0 & 0 \\
0 & 1 & 0 \\
0 & 0 & 0
\end{array}\right)\right)=0
$$

which gives, $-\lambda^{2}\left(2 \lambda p_{a}+\mu\right)=0$. The root $\lambda=0$ clearly corresponds to a repeated complex line-pair; $\alpha^{2}+\beta^{2}=0$. While the root $\mu=-2 \lambda p_{a}$ gives the real line-pair $\alpha \gamma=0$, that is the pair of lines $\alpha=0$ and $\gamma=0$.

Returning to the example, pitch quadrics restricted to the 3-system give the basis for the pencil:

$$
(\alpha, \beta, \gamma)\left(\begin{array}{ccc}
0 & 0 & 1 \\
0 & 0 & 0 \\
1 & 0 & 0
\end{array}\right)\left(\begin{array}{l}
\alpha \\
\beta \\
\gamma
\end{array}\right)=0 \text { and }(\alpha, \beta, \gamma)\left(\begin{array}{lll}
1 & 0 & 0 \\
0 & 1 & 0 \\
0 & 0 & 0
\end{array}\right)\left(\begin{array}{l}
\alpha \\
\beta \\
\gamma
\end{array}\right)=0 .
$$

These conics are clearly singular, again corresponding to the complex line-pair $\alpha^{2}+\beta^{2}=0$ and the real line-pair $\alpha \gamma=0$. Computing the characteristic 
equation for the pencil shows that the complex line-pair must be counted with multiplicity two. So this is indeed a $I B_{0}$ system and it is easy to see that the moduli must be $p_{a}=0$ and $p_{b}=0$. 


\section{Figure Captions}

Fig. 1 Body-fixed coordinate frame for a needle tip

Fig 2. Salkowski curves lying on quadrics. The left one has $m=0.42$ and the right has $m=1.0$. 